\title{
Efectos sorpresivos de la cláusula de la nación más favorecida (CNMF) en materia de inversiones extranjeras
}

(Estudio de la jurisprudencia del Centro Internacional de Arreglo de Diferencias relativas a las Inversiones -CIADI-)

The surprising effects of the most favored nation caluse (MFN) in regards to foreign investment (a study of the jurisprudence of the International Center for Settlement of Investment Disputes -ICSID-)

Recibido: 3 de octubre de 2008 - Revisado: 19 de octubre de 2008 - Aceptado: 7 de enero de 2009

Viviana Herrera Ramírez*

\section{Resumen}

La CNMF (Cláusula de la Nación más favorecida), insertada de forma casi automática en los tratados bilaterales de promoción y/o de protección de las inversiones durante las décadas de 1960 y 1970, devela hoy una dinámica sorprendente. Vía las decisiones de diferentes tribunales CIADI (Centro International de Arreglo de Diferencias relativas a Inversiones), presentamos la "evolución" o cambio que protagoniza esta cláusula.

Para esto, se analizará el mecanismo de la cláusula, el cual a priori circunscrito (por los Estados y la doctrina) a la importación de disposiciones de fondo, parece estar implícitamente habilitado para importar también disposiciones de procedimiento. La nueva faceta de la cláusula impone un estudio retrospectivo de sus principios, su enunciado y su objetivo. Mas dicha perspectiva no es el resultado de un cambio sustancial en el enunciado de la cláusula sino en la valoración que los árbitros, Estados e inversionistas hacen de ella. Así, la instrumentalización de la CNMF además de permitir una mayor liberalización del sector repercute en otras estructuras del derecho internacional y resalta el papel que desempeñan los Estados, sus cocontratantes privados y el CIADI en la relectura de la CNMF.

Palabras clave

CIADI, TBI (TPPI), CNMF, Arbitraje

\begin{abstract}
Nowadays, the Most Favored Nation (MFN) clause, which was incorporated almost automatically in Bilateral Investment Treaties (BIT) during the decades of 1960 and 1970, reveals a surprising dynamic. By means of different awards of the International Center for Settlement of Investment Disputes (ICSID) our article presents the "evolution" or change of conception that this clause has undergone. For this purpose it is necessary to analyze the clause mechanism, which a priori limited to importation of substantial provisions, seems to be implicitly authorized to insert procedural provisions too. The "new" feature of the MFN clause imposes a retrospective study of its principles, its wording and its aim. Such feature is not the consequence of modifications to the wording of the clause itself, but rather is the result of the assessments made by arbitrators, States and investors. That is why, the use of the MFN clause besides allowing a higher degree of liberalization in the investment sector, affects other structures of international law and highlights the role of States, investors and ICSID in the reinterpretation of MFN clause. Key Words ICSID, BIT, MFN, Arbitration.
\end{abstract}

\footnotetext{
* Abogada de la Universidad Sergio Arboleda, con maîtrise en Derecho Público y Maestría en Derecho Internacional de la Universidad Montesquieu Bordeaux IV (Francia). Actualmente doctorante de la misma institución en el área de Derecho Internacional.

Este trabajo es el resultado del desarrollo y de la continuación del tema de la monografía de maestría "Le régime de la Nation la Plus Favorisée en matière d'investissements internationaux à la lumière de la jurisprudence CIRDI".

Correo electrónico: vherreraramirez@yahoo.es
} 


\section{Introducción}

Durante los años 60 y 70, la CNMF fue incorporada de manera casi automática a los tratados de promoción y de protección de las inversiones (TPPI). Sin embargo, aunque anunciada siempre como la "CNMF", los términos que la definen son, raramente, los mismos.

En derecho internacional de las inversiones, por ejemplo se encuentran tantas CNMF como tratados PPI. Unas contemplan un campo de aplicación más extenso que otras, aunque todas garantizan a los inversores extranjeros igualdad de trato, es decir, la pluralidad de enunciados no impide la formación de un concepto jurídico único de TRATAMIENTO de la NMF, constituido por la obligación internacional que asume el Estado anfitrión.

La CNMF es esencialmente una excepción al derecho soberano de los Estados de establecer relaciones con los países que ellos quieran, cuando quieran y en los términos que quieran, limitando en el campo en que ha sido concedida, las negociaciones que posteriormente los Estados quieran adelantar. En el Caso relativo a los nacionales de los Estados Unidos en Marruecos, la Corte International de Justicia (CIJ) anunció que el propósito de esta disposición es "establecer y mantener en todo momento la igualdad fundamental sin discriminación entre todos los países interesados" (CIJ, 1952).

Por último, es evidente que en la práctica, la CNMF indica el grado de liberalización de un Estado determinado (Crépet, 2006; Leben, 2000): un país tendrá una política más liberal (en inversiones extranjeras) dependiendo de las facilidades consentidas para entrar y desarrollar actividades en su territorio.

\section{Liberalización del sector de las inversiones mediante la instrumentalización de la CNMF}

La CNMF permite al inversor beneficiario, el goce automático e inmediato de una ventaja, que habiéndole sido negada en el tratado de base (Estado Nacional-Estado anfitrión), fue concedida por el Estado anfitrión a un tercer Estado, eso sí independientemente de la naturaleza del instrumento en que fue concedida (acuerdo, acto legislativo unilateral, practica unilateral), mas ¿cuál es la naturaleza de dicha ventaja?, ¿puede una disposición de procedimiento ser importada vía la CNMF? A priori, esta cláusula interactúa con las disposiciones de fondo y nada prohíbe que lo haga con las disposiciones de procedimiento. Entonces, ¿cuáles son los límites de la CMNF? Éstos obedecen, unos a su naturaleza (principio ejusdem generis), y otros a su estructura (términos usados en la redacción). Veamos entonces la incidencia en el contencioso de los términos de la CNMF para examinar luego las precisiones hechas por CIJ y otras instancias internacionales.

\section{Límites convencionales de la CNMF}

Se utilizan diversos términos en la redacción de la CNMF, entre ellos:

1. La CNMF estipula, expresamente, su aplicación a las disposiciones relativas al arreglo de diferendos (Cláusula tipo de los TPPI del Reino Unido, Alemania y Países Bajos).

2. En la redacción de la CNMF, se usan términos "generales" que cubren, por ejemplo all matters, all rights, el traitment, pero que no hacen referencia alguna a las disposiciones relativas al arreglo de diferencias.

3. La CNMF está redactada en términos no generales, aunque guarda silencio en lo relativo a los mecanismos de arreglo de diferencias (NAFTA art. 3).

4. La CNMF expresa claramente que no se aplica a las disposiciones relativas al arreglo de diferencias (CAFTA, FTAA).

La primera y la última categoría no presentan mayores problemas. El árbitro debe aplicar, es 
decir, respetar la voluntad clara y expresa de las partes. En la tercera categoría, los términos específicos de la cláusula no permiten su extensión o generalización. La segunda categoría es, en cambio, fuente de incertidumbres. Cuando la CNMF está redactada en términos generales, es conveniente identificar los argumentos que permiten deducir que las partes querían excluir o incluir de su ámbito de aplicación, las disposiciones relativas al arreglo de diferencias.

Inicialmente, una CNMF que cubre all matters o el traitment y que no hace referencia a las disposiciones de procedimiento, se interpretaba como aplicable únicamente a las disposiciones de fondo. Así, durante mucho tiempo la atención se centró de forma casi exclusiva en los derechos sustantivos. Actualmente, el planteamiento de los diferendos cambia; los inversores quieren importar al tratado de base la cláusula de arreglo de diferencias (disposición procesual), que consentida por el Estado anfitrión, resulta más favorable a sus intereses.

La labor interpretativa de los árbitros está enmarcada por la Convención de Viena de 1969 (art. 31 y 32), instrumento que a pesar de contener reglas claras y concisas, no impide ni simplifica el contencioso originado alrededor de la interpretación de las cláusulas de la NMF. Si bien es cierto que "the main task of any tribunal which is asked to apply or construe or interpret a treaty... as the duty of giving effect to the expressed intention of the parties, that is, their intention as expressed in the word used by them in the light of the surrounding circumstances" (McNair citado en Vesel, 2001), cuando la CNMF es definida en términos ambiguios, el árbitro debe hacer una interpretación pro inclusión o pro exclusión de las cláusulas de arreglo de controversias.

Como se aprecia, la labor del árbitro no se reduce a la mera identificación de la obligación del Estado receptor del capital -del objetivo y contexto del TPPI en estudio y de la práctica de las partes- sino que incluye el análisis del rol de la CNMF y de las disposiciones de procedimiento [arreglo de diferendos] en el sistema del derecho de las inversiones.

Ahora bien, como es lógico, los inversores extranjeros buscan la aplicación de las garantías de tratamiento, generalmente contenidas en los tratados multilaterales, bilaterales o regionales de inversión. Sin embargo, las bondades de estas conquistas pueden reducirse a su mínima expresión, si dichos acuerdos no contemplan un mecanismo "adecuado" de arreglo de diferendos. He ahí la necesidad de prever un método alternativo y efectivo de solución de controversias que proporcione mayor seguridad a los inversionistas extranjeros. Es decir, tanto los inversores como los Estados buscan la protección de sus derechos y el desarrollo de sus actividades económicas en una atmósfera de confianza mutua, garantía que parece mejor protegida por el arbitraje internacional (UNCTAD, 2003).

La observación de los términos de las CNMF a la luz de las normas de interpretación, conduce a interpretarlos con miras a cumplir los objetivos de los acuerdos de inversiones. Es otras palabras, la generalidad de los términos all matters y traitment -introducidos en el texto de la CNMF- permitiría, salvo excepción expresa en contrario, la importación de la cláusula de arreglo de diferencias. La lectura del artículo 31(4) de la Convención de Viena (1969) -si las partes quieren dar una significación especial ,menos general- a las palabras introducidas en un tratado, tal intención debe constar en el instrumentocorrobora este punto de vista.

En definitiva, y de acuerdo con el aforismo nemo auditur propiam turpitudinem allegans, si las partes pudiendo formular una excepción a la CNMF relativa a la importación por esta vía de una cláusula de arbitraje o, pudiendo restringir el alcance de los términos (otorgándoles una significación especial) no lo hicieron, no corresponde al árbitro introducir tales excepciones o significados especiales.

Un razonamiento diferente indica que el mecanismo de la cláusula hace de ella una 
disposición de fondo, mientras que la cláusula de arreglo de diferencias es una regla de procedimiento. Siendo las dos de naturaleza diferente, no sería posible la importación de una regla de procedimiento mediante la utilización de una disposición sustantiva, pero la cláusula de arreglo de diferendos es, al mismo tiempo, parte del tratamiento concedido y garantizado por los Estados en los TPPI; es una garantía que constituye una incitación de fondo y una protección significativa (Gas Natural, ICSID case ARB/03/ 10). Desde este punto de vista, sería lógico sostener que el inversor beneficiario puede favorecerse, mediante la CNMF con una cláusula de arreglo de diferencias más favorable. Es más, de considerarse que el acceso a un tribunal arbitral internacional es la concesión más importante otorgada por un TBI (garantía de la efectividad de las disposiciones sustantivas), sería contradictorio excluir esta garantía del ámbito de la CNMF.

Luego de analizar el alcance textual de la CNMF veamos sus límites rationae materiae.

Precisiones de la CIJ al mecanismo de la CNMF

- La CIJ en el análisis del caso Anglo Iranian Oil Company (1952) recordó que la CNMF contiene un compromiso cuyo objeto es real, aunque su contenido no es determinado y es susceptible de cambiar de volumen dependiendo de los tratados concluidos con terceros. La aplicación de esta decisión a las inversiones extranjeras representaría la situación de un demandante, parte de un TBI que no contiene cláusula de arbitraje, que busca acceder a través de la CNMF a un tercer tratado que contiene dicha cláusula. ¿Debe el Tribunal Arbitral constatar su falta de competencia o puede, bajo ciertas condiciones, estudiar el caso?

- La duración del beneficio importado vía la CNMF fue estudiada en el caso derechos de los nacionales de Estados
Unidos en Marruecos (CIJ, 1952). En éste se trataba de determinar si los privilegios de que gozaba el Estado beneficiario de la CNMF (Estados Unidos) se incorporaban de forma autónoma al tratado de base, de manera que la renuncia [a los favores] pronunciada por los terceros favorecidos (Gran Bretaña y España) debía ser interpretada como res inter alios acta. $\mathrm{O}$, si una vez pronunciada dicha renuncia, el Estado beneficiario podía reclamar los privilegios a título de la costumbre. La Corte rechaza la petición de los Estados Unidos afirmando que tal pretensión va en contra de la función de la CNMF: establecer y mantener, en todo tiempo, la igualdad fundamental sin discriminaciones entre todos los países interesados.

Recuerde, rápidamente, el funcionamiento de la cláusula: la CNMF concede al Estado beneficiario un derecho autónomo e independiente cuyo contenido depende del favor que sea reconocido a una tercera Nación (Estado favorecido). Una vez el Estado favorecido renuncia al favor, el contenido del derecho del Estado beneficiario se disipa. La desaparición de la ventaja configura entonces, la inexistencia del hecho que determina la entrada en vigor de la cláusula: cessante causa, cessat effectus (Snyder, 1948 citado en Ustor, 1974). Es decir, mientras el derecho a recibir un trato no menos favorable que el otorgado a la nación más favorecida resta intacto, su contenido concreto desaparece, respetándose así el principio de igualdad, eje principal de la CNMF.

- La CIJ y una comisión de arbitraje precisaron el principio ejusdem generis en su estudio del caso Ambatielos (1952). "En virtud de la CNMF el Estado beneficiario adquiere únicamente los derechos que están dentro de los límites de la materia que es objeto de la cláusula" (CDI, 1978) (traducción del autor). La 
comisión de arbitraje agrega que la CNMF no podrá extenderse a materias diferentes a aquellas por las cuales ha sido estipulada $\mathrm{y}$ concluye que "it is true that "the administration of justice", when viewed in isolation, is a subjectmatter other than "commerce and navigation", but this is not necessarily so when it is viewed in connection with the protection of the rights of traders (...) Therefore it cannot be said that the administration of justice, in so far as it is concerned with the protection of these rights, must necessarily be excluded from the field of application of the mostfavourednation clause, when the latter includes "all matters relating to commerce and navigation"” (p. 107).

La lectura extensiva de esta conclusión soporta la idea de que una CNMF redactada en términos generales (all matters), incorpora, salvo estipulación expresa en contrario, las cláusulas de arreglo de diferencias. Esta fue la posición adoptada por los Tribunales CIADI que conocieron de los casos Maffezini, Siemens, Tecmed y Camuzzi. Mientras que los Tribunales Salini, Plama, Telenor [y Wintershall] defendieron la tesis contraria. Examinemos ahora los argumentos expuestos en estas decisiones, buscando identificar la regla o el principio que define el alcance de la CNMF.

Establecimiento de una regla: la aplicación de la CNMF a las disposiciones relativas al arreglo de diferencias

Al comienzo, el mecanismo de la CNMF fue circunscrito - por una parte de la doctrina y de Estados- a las garantías sustanciales. Actualmente algunos autores consideran que la CNMF tiene un mayor alcance: su aplicación cubriría las reglas de procedimiento -cláusulas de arreglo de diferencias: reconocimiento, ejecución y anulación de los laudos- (Ben Hamida, 2003; see also Gaillard, 2006; Sutton, 2005).
Sobre el "presumido" vasto alcance de la CNMF, Gaillard hace una precisión (2007); para él, y contrariamente a lo que puede a veces escucharse, todas las disposiciones de un tratado no son susceptibles de ser afectadas por el juego de la CNMF. La inversión que no cumple con los requisitos de aplicación del tratado (noción de inversión, las eventuales exigencias de nacionalidad por ejemplo), no puede beneficiar del régimen allí contemplado ni, por tanto, de su CNMF. En contraste con lo que una visión puramente procedimental puede aseverar, continúa el autor, la puerta de entrada de un tratado son las cláusulas que definen su campo de aplicación y no la cláusula de arreglo de controversias. El cumplimiento de los elementos de aplicación del tratado constituye el requisito sine qua non la protección del tratado no puede solicitarse, poco importa que otros tratados contengan diferentes requisitos o que éstos sean más flexibles.

Con base en el punto de partida, se estudiarán estas ideas en el aparente principio de aplicación de la CNMF a las reglas de arreglo de diferendos.

\section{Regla proferida en el caso Maffezini (ICSID Case No. ARB/97/7)}

En 1997, el inversor argentino Emilio A. Maffezini afirma ante un tribunal CIADI, que las acciones de ciertos organismos españoles causaron daños a sus inversiones en una empresa productora y distribuidora de productos químicos, ubicada en Galicia, configurándose entonces una violación del TPPI España y Argentina (3 de octubre de 1991). En su demanda Maffezini invocó el artículo 10 -que permite que un diferendo sea llevado ante un tribunal arbitral cuando, después de una etapa de negociación de 18 meses, los tribunales internos no han adoptado una decisión de fondo-, y el artículo 4.2 (CNMF) del TBI para beneficiar del TBI España-Chile.

El tribunal arbitral estudia el artículo 10 y concluye que el demandante no ha cumplido con los requisitos de este artículo (el transcurso de 
los 18 meses); por consiguiente el consentimiento al arbitraje pronunciado por España no se verificaba (36). Sin embargo, la cláusula de arreglo de diferencias del tratado España-Chile permitía al inversor acudir a un tribunal arbitral al final de los 6 meses previstos para la negociación entre las partes.

El tribunal, después de estudiar las decisiones pronunciadas sobre la materia por la CIJ y por otros tribunales CIADI (42-51), de determinar el tratado de base, de examinar el principio ejusdem generis (46-49) y de revisar la intención y práctica (52-54 y 58-61) de las partes, concluye que no permitir a los inversores el acceso a la cláusula de arreglo de diferencias que les es más favorable, no es coherente ni con el enunciado de la cláusula, cuya redacción no limita su aplicación a las disposiciones subjetivas, ni con su objetivo: la armonización de los sistemas de tratamiento de las inversiones extranjeras.

A este respecto, consideramos importante resaltar, que a diferencia de este tribunal y de Crépet (2006) no vemos cómo, tratándose de las cláusulas de solución de controversias, tal armonización puede alcanzarse gracias al juego de la CNMF. A nuestro juicio, la armonización del sistema obedece más a la modelización del Derecho Internacional de Inversiones que a la inclusión de la CNMF.

En cuanto al texto de la decisión, el cual retomamos, luego de resaltar que el arbitraje es esencial para proteger los derechos previstos en los tratados y está estrechamente vinculado con los aspectos sustantivos del tratamiento acordado (55), el Tribunal hace una afirmación sorprendente, según la cual, "no obstante el hecho de que el tratado básico que contiene la cláusula no se refiere expresamente a la solución de controversias como una materia cubierta por la cláusula $[\mathrm{NMF}](\ldots)$ hay razones suficientes para concluir que actualmente los arreglos relativos a la solución de controversias están inseparablemente vinculados con la protección de inversionistas extranjeros, como también se vinculan con el resguardo de los derechos de los comerciantes en los Tratados de Comercio" (54). Sin hacer otras precisiones ni sugerir o indicar cuáles son esas "razones suficientes", el Tribunal llega a la conclusión.

Con estos términos es importada al Derecho Internacional de Inversiones la tesis según la cual las disposiciones de procedimiento son en principio evocables vía la CNMF: “ (...) si un Tratado con un tercero contiene disposiciones para la solución de controversias que sean más favorables para la protección de los derechos e intereses del inversor, que aquellos del Tratado básico, tales disposiciones pueden extenderse al beneficiario de la cláusula de la Nación más favorecida, pues son plenamente compatibles con el principio ejusdem generis (56)".

El contenido y la generalidad de las afirmaciones del Tribunal Maffezini podrían interpretarse como la voluntad de establecer un principio, aunque las sutiles, pero importantes diferencias que caracterizan las cláusulas NMP ¿permiten realmente la formulación de un principio?

\section{Consecuencias y excepciones a la "regla"}

Conscientes de la extensión de sus afirmaciones, el Tribunal Maffezini advierte que la aplicación de la CNMF "a los arreglos de solución de controversias en el contexto de los tratados sobre inversiones (...) no [puede] dejar sin efecto las consideraciones de política pública que puedan haber previsto las partes contratantes como condiciones fundamentales [de] su aceptación $(\ldots) "(62)$.

Según el tribunal hacen parte de estas consideraciones: la exigencia del agotamiento de los recursos internos (condición prevista en el Convenio del CIADI: art. 26); la observación de la cláusula de "bifurcación del camino" (La decisión de someterse a determinada jurisdicción es "definitiva e irrevocable [y] no puede ignorarse 
mediante la invocación de la cláusula [CNMF]); la designación de una institución arbitral específica; y el acuerdo de someterse a un sistema de arbitraje altamente institucionalizado que incorpore reglas de procedimiento precisas (63). Como esta enumeración es a título indicativo, el Tribunal añade que para identificar una situación de política pública, es necesario saber diferenciar "entre la legítima extensión de derechos y beneficios mediante la aplicación de la cláusula, por una parte, y la alternativa nociva de tratar de aplicar diversos tratados para alterar los objetivos de política en que se fundamentan algunas disposiciones específicas, por la otra" (63).

Consideramos, al igual que Dolzer y Myers (2004), que las tres últimas excepciones presentadas en Maffezini, están más relacionadas con el respeto de la voluntad explícita y común de las partes que con argumentos de política pública. A nuestro parecer, dichas excepciones reflejan el principio lex specialis derogat legem generalem. En consecuencia la CNMF, disposición general, no puede desconocer las estipulaciones contractuales específicamente convenidas entre las partes.

Los efectos de la decisión Maffezini no se hicieron esperar. Los Estados en cuyos Acuerdos Bilaterales de Inversión (ABI, TBI o TPPI), la voluntad de incluir o excluir el arreglo de las controversias en el campo de aplicación de la CNMF era ambigua, entonces introdujeron anotaciones específicas al respecto (FTAA, 2003). Los inversores, por su parte, comenzaron a evaluar, minuciosamente, los tratados suscritos por el Estado anfitrión para determinar e invocar la cláusula de arreglo de controversias que les fuera más favorable.

A manera de conclusión queremos resaltar que una corriente doctrinal importante (Crépet, 2006; Dolzer, 2004; Gaillard, Establishing, 2005), cree que tanto el principio como las excepciones enunciadas en Maffezini son de difícil aplicación y que la reacción de los Estados frente a esta decisión ha sido juzgada por unos como la confirmación, y por otros, como el rechazo al "principio" enunciado en Maffezini (Poulain, 2007).

\section{Inexistencia de una regla: la particularidad del laudo Maffezini}

La especificidad de las pretensiones y la particularidad de las disposiciones estudiadas en Maffezini impiden que esta decisión se convierta en regla o principio interpretativo.

\section{Aplicación de la CNMF a las condiciones de admisión de la demanda}

En el caso Maffezini el demandante buscaba beneficiarse de las condiciones más favorables de admisión de la demanda que estaban contenidas en un tercer tratado. El mismo problema y planteamiento fue recreado en controversias posteriores, las cuales se analizarán brevemente.

Siemens (ICSID Case No. ARB/02/8). Un inversor alemán buscaba ignorar la condición de acudir a los Tribunales argentinos y esperar que transcurrieran 18 meses antes de acudir a un Tribunal arbitral (TBI Alemania-Argentina), con el fin de acceder, vía la CNMF, a la cláusula de arreglo de diferendos del Tratado Chile-Argentina (1991) que contemplaba un período de negociación de tan solo 6 meses.

Cabe resaltar la diferencia de términos empleados en el texto de las CNMF. En el Tratado Alemania-Argentina, la CNMF no es tan general como la analizada en Maffezini, ésta última se aplica a "todas las cuestiones sujetas a este acuerdo", mientras que la primera sólo al "trato". El Tribunal arbitral toma nota de la diferencia, pero concluye que las dos expresiones son lo suficientemente generales para incluir el arreglo de diferencias.

El Tribunal Siemens elabora su conclusión en tres etapas. Primero, resalta la importancia del arbitraje internacional para la protección y 
promoción de las inversiones (102). Segundo, rechaza la afirmación del Gobierno argentino, según la cual la regla de agotamiento de los recursos locales, además de ser un elemento esencial de su consentimiento al arbitraje, está íntimamente vinculada a cuestiones de política económica y exterior $(104,105)$. Tercero, niega que la reclamación de un beneficio en virtud de una CNMF desencadene la aplicación de la totalidad del tratado $(108,109$ y 120$)$.

Aún más, aunque consciente de que las ventajas concedidas en un tratado corresponden, en cierto modo, a las cargas asumidas, el Tribunal no hace referencia alguna a la cláusula de bifurcación de vías que limita la CNMF en el Tratado Argentina-Chile (que no está presente en el Tratado Alemania-Argentina). De esta forma, al afirmar que el inversor alemán puede, sin observar la regla de bifurcación del camino, acudir directamente al CIADI, el tribunal impone a Argentina una combinación de cláusulas de arreglo de controversias que ésta -tal vez- nunca hubiese aceptado. Finalmente, si el Tribunal compartía la teoría expuesta en Mafezzini, ¿por qué no invocó la segunda excepción allí inscrita? ¿Cuál fue su racionamiento?

Otras decisiones confirman la posición del Tribunal Maffezini. En Gas Natural (ICSID Case No. ARB/03/10) el Tribunal advierte que el arbitraje internacional hace parte de la protección brindada por los TBI (31) y que los términos generales de la CNMF permiten concluir que los Estados tenían la intención de incorporar, en su ámbito de aplicación, la cláusula de arreglo de controversias. No obstante, no se hace ninguna referencia al caso Siemens, en el cual la presencia de una CNMF redactada en términos menos generales no fue óbice para considerar que el arreglo de las controversias hacía parte del ámbito de aplicación de la CNMF.

Hechos similares fueron estudiados en el caso Camuzzi (ICSID Case No. ARB/03/7): el demandante (Belga-Luxemburgués) beneficiándose de la cláusula de arreglo de controversias de un tercer tratado, (Estados Unidos -Argentina del 14 de noviembre de 1994), acude directamente ante un tribunal arbitral sin haber llevado el litigio ante las autoridades argentinas, tal como lo exigía el Tratado de base. El Tribunal no argumenta su decisión sobre este punto, ya que Argentina había aceptado (no contestó), la jurisprudencia Maffezini.

\section{Aplicación de la CNMF para beneficiar, retroactivamente, una disposición}

En el caso TECMED (ICSID Case No. ARB (AF)/00/2), un inversor español deseaba que el comportamiento del Estado mexicano anterior a la entrada en vigor del Tratado [México - España del 18 de diciembre de 1996] fuera sometido a las obligaciones del Tratado. Buscando paliar la no retroactividad del tratado de base, el demandante utiliza la CNMF para invocar los artículos 27 (el tratado es aplicable a las inversiones constituidas antes de su entrada en vigor, pero no a las demandas interpuestas antes de aquella fecha) y 11 del Tratado MéxicoAustria.

El Tribunal rechaza las pretensiones del demandante argumentando que las "cuestiones vinculadas a la aplicación en el tiempo del acuerdo, que en realidad conciernen más al ámbito temporal de aplicación de sus disposiciones sustantivas, que a cuestiones de índole procesal o jurisdiccional, por su trascendencia e importancia, integran el núcleo de cuestiones que deben presumirse como especialmente negociadas entre las partes contratantes y determinantes de su aceptación" (69, negrilla fuera del texto).

Las críticas doctrinales han sido diferentes, por lo cual han recibido esta decisión. Crépet Daigremont (2006) piensa que, contrariamente a lo que deja entrever el Tribunal TECMED, todas las disposiciones de un tratado son negociadas especialmente por las partes. A su juicio la verdadera razón que impide la aplicación, en este contexto, de la CNMF es que la aplicación en el tiempo del tratado concierne la vida de este 
instrumento y no el contenido del tratamiento acordado con los inversores [o inversiones]. La CNMF permite atraer un trato más favorable, pero la retroactividad de un tratado no constituye una ventaja y no puede, por tanto ser importada vía la CNMF. Gaillard (2006) afirma exactamente lo contrario. Para este autor, por ejemplo las cláusulas de arbitraje reflejan la política económica de los Estados y por este hecho son heredadas de un acuerdo al otro, sin que medie una negociación específica. En consecuencia, cuando un Estado ligado previamente por TBI donde ha concedido la CNMF, adopta una política más liberal (acepta el arbitraje como mecanismo de solución de controversias), reconoce obligatoriamente la aplicación de esta política a todos los otros Estados que han suscrito un TBI con él.

Por su parte, S. Fietta (2005) ve en la referencia hecha a "las disposiciones especialmente negociadas" el primer signo de un movimiento hacia la limitación de la jurisprudencia Maffezini (movimiento que se concretizará con la decisión Plama). R. Dolzer y T. Myers (2004), creen en cambio que esta decisión es un addendum a las excepciones enunciadas en Maffezini. La nueva excepción permite que otras materias sean identificadas como "específicamente negociadas".

Estos argumentos llevan a hacer dos precisiones. Afirmar que ninguna disposición de un Tratado puede ser desconocida vía la CNMF, ya que todas han sido específicamente negociadas, desconoce, de manera abierta, la intención de las partes: dotar de efecto la CNMF. Y, de admitirse que la frase "disposiciones específicamente negociadas" constituye un límite válido a la aplicación de la CNMF, sería pertinente identificar cuáles son las disposiciones que son "específicamente negociadas". Ahora bien, ¿Qué criterios permitirían determinar tales disposiciones?

\section{Aplicación de la CNMF para extender la competencia de los Tribunales}

La lectura hecha por Tribunal Maffezini de una CNMF que no precisa su ámbito de aplicación preocupa a una parte de la Comunidad Internacional: la decisión parece señalar una supuesta autonomía y jerarquía entre las disposiciones de los TBI, por tanto los Estados resaltan las incertidumbres convencionales y la inseguridad jurídica que se deriva de dicha interpretación, mientras que los inversores aplican con mucha astucia la cláusula. Con seguridad, éstas fueron algunas de las ideas desarrolladas en los laudos que rechazaron, total o parcialmente, la doctrina Maffezini.

El Tribunal CIADI que conoció del caso Salini (ARB/02/13) estudió las relaciones existentes entre las diferentes disposiciones de un TBI y, entre éstas y una cláusula de arreglo de diferencias contractuales. En este caso, el demandante italiano buscaba dejar sin efecto la cláusula de arreglo de diferencias estipuladas en el contrato de concesión -cláusula que de acuerdo con el TBI Italia-Jordania (1999) debía ser aplicada en caso de conflicto-, y solicitaba la aplicación del artículo X del TBI Jordania-USA ( 2 de julio de 1997), y/o del artículo 6 du TBI Jordania-Reino Unido (10 de octubre de 1979), los cuales permitían llevar ante el CIADI todo diferendo relativo a un contrato de construcción.

Según su criterio concluye, luego de advertir que la decisión Maffezini, además de ser de difícil aplicación, aumenta el riesgo de treaty shopping; el Tribunal destaca que las partes al TBI de base habían manifestado expresamente la voluntad (artículo 9.2) de someter a los mecanismos de solución previstos en el contrato todos los litigios originados por la ejecución de éstos (Lex specialis derogat legem generalem). El Tribunal concluye entonces, que la CNMF (art. 3 TBI ItaliaJordania) no cubría las disposiciones relativas al arreglo de las controversias.

Es interesante observar que el Tribunal Salini pudo haber llegado a la misma conclusión invocando la excepción de política pública (jurisprudencia Maffezini). Además, hubiese sido enriquecedor saber qué argumentos podría haber utilizado el Tribunal para justificar la solución 
contraria adoptada en Siemens: los términos de las CNMF estudiadas en Salini y Siemens, no eran tan amplios como aquellos de la CNMF de Maffezini, ni la demandante había probado que la voluntad de las partes al Tratado de base era aplicar la CNMF a las disposiciones de procedimiento. Finalmente, aunque el Tribunal se declara no competente, su conclusión parece fundarse más en la ausencia de elementos que permitan adherir a la jurisprudencia Maffezini que en la naturaleza misma de la CNMF.

Otros Tribunales siguen la línea trazada en Salini. En el caso Plama (ICSID Case No. ARB/03/04), el Tribunal CIADI examina el ámbito de aplicación de la CNMF dentro del marco del Tratado de la Carta de la Energía (parte V: disposiciones relativas al arreglo de las controversias, art. 26). Como la cláusula de solución de controversias del TBI de base (Bulgaria-Chipre, 1987) permitía recurrir solamente a un arbitraje ad-hoc según el reglamento UNCITRAL y limitaba negocios solucionables por esta vía, estaban limitados a la indemnización en caso de expropiación juzgadailegal por los Tribunales del país anfitrión; la demandante invocó la CNMF (art.3) para poder acudir al CIADI (cláusula de arreglo de controversias concedida por Bulgaria a terceros países).

En su decisión el Tribunal al mismo tiempo que resaltó la importancia actual del arbitraje, recordó que "that phenomenon does not take away the basic prerequisite for arbitration: an agreement of the parties to arbitrate. It is a well-established principle, both in domestic and international law, that such an agreement should be clear and unambiguous" (198). Resulta de esto que una CNMF "in a basic treaty does not incorporate by reference dispute settlement provisions in whole or in part set forth in another treaty, unless the MFN provision in the basic treaty leaves no doubt that the Contracting Parties intended to incorporate them"(223) (negrillas fuera del texto). Es decir, el principio enunciado en Maffezini se convierte en la única excepción a la regla de la no aplicación de la CNMF a las disposiciones de procedimiento (cláusula de arbitraje).

En Plama y en Salini, la CNMF era invocada para importar al Tratado de base el consentimiento del Estado anfitrión al arbitraje CIADI, diferencia inconmensurable con el caso Maffezini ya que "it is one thing to add to the treatment provided in one treaty more favorable treatment provided elsewhere. It is quite another thing to replace a procedure specifically negotiated by parties with an entirely different mechanism" (Plama 209).

Al igual que en el caso presentado anteriormente, es conveniente señalar que el Tribunal pudo haber llegado a la misma conclusión siguiendo la jurisprudencia Maffezini, es decir, invocando la excepción de política pública. Tal dinámica hubiese tenido, al menos, el mérito de consolidar la jurisprudencia; sin embargo, los árbitros prefirieron resaltar que las circunstancias analizadas en Maffezini son excepcionales, por ende no pueden “(...) be treated as a statement of general principle guiding future tribunals in other cases where exceptional circumstances are not present» (224) (negrilla fuera del texto).

Otra decisión en el mismo sentido, pero más vigorosa fue adoptada en el caso Telenor (ICISD Case No. ARB/04/15). Una sociedad de derecho noruego, basándose en el TBI NoruegaHungría (8 de abril de 1991), acude al CIADI para que se resuelva una controversia originada en un contrato de concesión de servicios de radiotelefonía pública móvil. De un lado, el contrato estaba sometido al derecho húngaro y atribuía la competencia a las autoridades húngaras, y del otro, el artículo XI del TBI limitaba la competencia del tribunal CIADI al contencioso de la expropiación, de la repatriación de capitales y a la indemnización debida en caso de destrucción de la inversión por guerra, revolución o eventos análogos. Por estas razones y para acceder a cláusulas de solución de controversias [juzgadas más favorables] acordadas por Hungría a terceros Estados la demandante invoca la CNMF del TBI de base (artículo IV). 
El Tribunal analiza la jurisprudencia y concluye que "the scope of the Tribunal's jurisdiction is limited by Article XI to claims involving expropriation and that the MFN clause embodied in Article IV cannot be invoked to extend the Tribunal's jurisdiction to claims under Article III" (Conclusiones).

En consecuencia, el Tribunal afirma que hay al menos cuatro razones por las cuales una CNMF no puede ser invocada [salvo si ella lo precisa expresamente] cuando la cláusula de arreglo de diferendos limita la competencia del tribunal arbitral a cierto tipo de contenciosos. En primer lugar, de acuerdo con la Convención de Viena sobre el Derecho de los Tratados, el sentido ordinario de la expresión "los inversores deberán acordar un trato no menos favorable que el otorgado a las inversiones de inversores de cualquier otro Estado", hace referencia a los derechos sustantivos y no debe ser interpretada como importando los derechos de procedimiento. Segundo, los riesgos de treaty shopping. Tercero, la extensión del ámbito de aplicación de la CNMF genera inseguridad e imprevisibilidad: una cláusula de arreglo de las controversias limitada es, en un premier tiempo, efectiva pero vía la CNMF puede ser transgredida el día que el Estado anfitrión conceda una disposición menos o no limitada. Cuarto, el árbitro debe interpretar la voluntad de las partes y no remplazar, por consideraciones de política general de protección de las inversiones, los mecanismos de solución de controversias específicamente negociados por las partes (92-95) (traducido por el autor).

El remarcable esfuerzo del Tribunal Telenor de neutralizar el riesgo de dilución del consentimiento al arbitraje fue fuertemente criticado por Gaillard (2006), quien entre otras cosas subrayó que, la afirmación según la cual el juego de la CNMF aumenta el riesgo de treaty shopping desconoce la naturaleza de esta cláusula; que, el argumento relativo a la inseguridad jurídica corresponde a una visión en extremo estática del derecho internacional, visión que aplicada a las disposiciones de fondo, conduciría a la inexistencia de la CNMF; y que no se desconoce la voluntad de las partes cuando se aplica la CNMF, ya que la intención de las partes es que ésta surta efecto.

Es importante recordar que el árbitro debe tener siempre presente los intereses de las partes en conflicto. No se trata de interpretar las cláusulas a favor del inversor, ni a favor del Estado, sino de establecer cuáles fueron las garantías otorgadas al inversor y cuáles son sus límites. Para lograr esto es necesario sopesar el objetivo de la CNMF y de los TBI, sin dejar de lado la voluntad explícita e implícita de las partes. En Maffezini el justo medio fue encarnado por la excepción de política pública, entonces, ¿es ésta la margen correcta? ¿Qué disposiciones hacen parte de ella ["política pública"]?

Es pertinente presentar paralelamente el laudo RosInvest Co. UK Ltd c/ La Federación Rusa (octubre de 2007), proferido por el Tribunal Arbitral de la Cámara de Comercio de Estocolmo. En este negocio el demandado quería mediante la CNMF (art. 3 TPI Unión Soviética-Reino Unido, 1989), eludir la aplicación del artículo 8 del TBI de base (que limitaba la competencia del Tribunal Arbitral a la fijación del monto o pago de la indemnización y a las cuestiones relativas a la expropiación) para beneficiar en cambio del de la cláusula de arbitraje del TPPI DinamarcaRusia, que no limitaba rationae materiae la competencia del Tribunal Arbitral.

En esta ocasión, y a diferencia de la decisión Telenor, el Tribunal acepta la utilización de la CNMF que extiende su competencia-rationae materiae-. Los argumentos son: primero, el mecanismo de la cláusula permite que una ventaja negada en un TPPI y concedida en otro, sea incorporada en el primero (132); y segundo, los Estados partes al TPPI de base enumeraron en el artículo 7 las excepciones aplicables a la CNMF. Ahora bien, como las cláusulas de arbitraje no se encuentran entre ellas, puede concluirse que la CNMF cubre las cláusulas de arbitraje (135). Este último argumento distingue 
el caso sub judice de las circunstancias estudiadas en Telenor y permite deducir de forma más "certera" que la voluntad de las partes no era excluir del ámbito de la CNMF la cláusula de arbitraje.

La importancia de esta decisión se resume en que, a pesar de que dentro de sus circunstancias particulares, no busca imponer una teoría definitiva a propósito de la importación vía la CNMF de las disposiciones de procedimiento, parece dar un paso en la misma dirección de Maffezini. No obstante, es necesario aclarar y subrayar que el Tribunal extendió su competencia material, pero no se autootorgó la jurisdicción ni la importó de un tercer Tratado: en el Tratado de base, las partes habían clara y expresamente acordado que las controversias que sugieran serían resueltas por el Tribunal de Arbitraje de la Cámara de Comercio de Estocolmo.

Volviendo al CIADI, encontramos dos decisiones de diciembre de 2008 que se apartan decisión Maffezini: Wintershall Ag c/ Argentina (Case No. ARB/04/14) y TSA Spectrum de Argentina c. Argentina (Case No. ARB/05/5). La extensión del artículo impide analizar en detalle las dos decisiones, a continuación estudiaremos sólo la primera de ellas.

Wintershall es una sociedad alemana que realizó inversiones en el sector argentino de la producción de petróleo y gas natural. Según esta sociedad, la legislación de emergencia (adoptada para hacer frente a la crisis económica argentina) violó varias de las obligaciones contenidas en el TPPI Argentina-Alemania. Ya que durante el período de negociación amigable (6 meses) no se llegó a ningún acuerdo, Wintershall decide someter el conflicto a un tribunal CIADI. Para fundar la jurisdicción del Tribunal la demandante invoca, a través de la CNMF (art. 3 del TPPI de base), la cláusula de arbitraje del TPPI ArgentinaEE.UU (art. VII); disposición que a diferencia de la cláusula de arbitraje del TPPI de base (art.10), no exigía como etapa previa a la competencia del CIADI, el sometimiento de la controversia a los tribunales argentinos.
El tribunal estudia la jurisprudencia sobre la materia y critica fuertemente la presunción establecida en Siemens. Para este tribunal la conclusión que permite "deducir" que el "trato" acordado a los inversores (término no general) incluye las disposiciones procesuales "tends to create meaning rather than to discover it". Incluso, el Tribunal agrega que "even words like "all matters relating to (...)" [términos generales] in an MFN clause may not be sufficient to extend such clause to the dispute resolution provisions of the BIT" (185 y 186).

Después de observar la "jurisprudencia" general en la materia (incluido el caso National Grid c. Argentina donde el demandante consiguió gracias a la aplicación de la CNMF evadir el requisito previsto en el TPPI de base, según el cual la controversia debía ser llevada ante los tribunales internos, antes de acceder al arbitraje), el Tribunal concluye que de acuerdo con los términos del art. 10 del TPPI de base, el derecho a iniciar un arbitraje ante el CIADI está expresamente condicionado al previo sometimiento del conflicto a los tribunales argentinos competentes (durante un período de 18 meses) (ver artículo 26 Convención CIADI); por ende, la aplicación de la CNMF no es viable.

La lectura de esta decisión nos llama la atención sobre dos aspectos. Primero, a diferencia de lo arguiido por la demandante, el Tribunal considera que la aplicación de la CNMF a las cláusulas de solución de diferendos afecta la jurisdicción, el consentimiento al arbitraje e incluso el mecanismo de solución de las controversias. Y, Segundo, el tribunal recuerda que "the obligation "to submit to arbitration cannot be enlarged by interpretation"", y "a jurisdictional clause must on no account be interpreted in such a way as to exceed the intention of the States that subscribed it" (161). El Tribunal es claro al hacer sus observaciones. Él confirma tajantemente la conclusión proferida en Plama (la CNMF no es aplicable a la cláusula de arbitraje, salvo la voluntad expresa de las partes) $\mathrm{y}$, frente a las diferentes teorías (decisiones) sobre 
el ámbito de aplicación de la CNMF, cita un libro (Inter-national Investment Arbitration: Substantive Principles) que presenta de manera brillante la situación: "As the ICJ pointed out -in East Timor (Portugal) v. Australia-, the scope of application of a substantive obligation is an entirely separate question to the conferral of jurisdiction upon an international tribunal, Jurisdiction in International law depends solely upon consent. This is a difficult concept in any event in investment arbitration. Given the absence of a meeting of minds between investor and host State, consent has to be constructed from the standing consent given by the State by treaty, and the subsequent consent given by the investor at the time the claim is submitted to arbitration. In those circumstances, it is particularly important to construe the ambit of the State's consent strictly." (Shore y Weiniger citados en Wintershall 188).

Como se ha visto a lo largo de este artículo, la CNMF es uno de los protagonistas del desarrollo del Derecho Internacional de las Inversiones. Su mecanismo relacional ha mostrado algunos de sus efectos, pero posiblemente su naturaleza nos reserve aún más sorpresas.

\section{Conclusiones}

La interpretación de la CNMF en Derecho Internacional de las Inversiones, se ha transformado en un punto neurálgico que gira sobre la teoría de su extensión o no a las cláusulas de procedimiento.

Los Tribunales CIRDI, en ciertos negocios, han incorporado la cláusula de arbitraje en el ámbito de aplicación de la CNMF; y en otros, han afirmado tajantemente lo contrario. Estas decisiones a priori contradictorias, se inscriben en contextos diferentes y son el resultado de CNMF redactadas diferentemente. Así la heterogeneidad de los elementos que deben ser analizados y la complejidad de las relaciones internacionales demandan posiciones más flexibles y constituyen el principal óbice a la formación de una regla o principio.

En este contexto se destacan dos posiciones. Una, asevera que ante la ambigüedad de la CNMF, el objetivo de establecer un ambiente de confianza mutua y la igualdad de los inversores debe prevalecer y guiar la labor interpretativa de los árbitros. La otra, sin desconocer los objetivos de los TPPI, aduce que la aplicación de la CNMF no puede transgredir los principios básicos del Derecho Internacional, ni imponer a las partes a un Tratado elementos que ellas no habían previsto, ni mucho menos alterar la estabilidad del Derecho Internacional (Vesel, 2001).

A nuestro parecer sólo el examen detallado de cada CNMF [de su contexto] y el respeto de las condiciones fijadas por los Estados como requisito previo al arbitraje permitirán mantener la legitimidad del sistema CIADI; sistema, que es bueno recordarlo, no se estructura alrededor de los precedentes y no contempla el recurso de apelación.

De igual manera, queremos subrayar que en todo caso [apriorísticamente] debe ser eliminada la posibilidad de importar al tratado de base el consentimiento al arbitraje dado a un Centro determinado (CIADI por ejemplo), que aunque concedido en un tercer Tratado brilla por su ausencia en el Tratado de base. El principio que subordina la jurisdicción de los tribunales arbitrales al consentimiento de las partes "should [not] be held to have been tacitly dispensed, in absence of any words making clear an intention to do so", es decir, no puede ser establecido a través de la CNMF.

Ahora bien, teniendo en cuenta que el consentimiento al arbitraje determina dos aspectos: la jurisdicción y la competencia de dicha jurisdicción, puede considerarse viable la posibilidad de que la competencia del CIADI, limitada al conocimiento de conocer cierto tipo de negocios [tratado de base], pueda verse 
ampliada gracias al juego de la CNMF. Esto únicamente en el evento en que la cláusula de arbitraje que no limita la competencia del CIADI, sea el resultado del cambio de orientación política del Estado anfitrión (ver Gaillard, 2006). Continuando con esta idea, aun cuando el arbitraje sea una garantía importante para el inversor, ésta no puede ser reconocida por el árbitro mediante el ejercicio inapropiado de su jurisdicción. Sería inadmisible que un Tribunal arbitral se auto-otorgue jurisdicción en detrimento de otro Tribunal arbitral: el consentimiento al arbitraje de la Cámara de Comercio de Estocolmo, por ejemplo no puede ser constitutivo del consentimiento al arbitraje CIADI. ¿Qué argumentos permitirían al CIADI afirmar que su jurisdicción es más favorable que la de otros centros de arbitraje?

Los intereses en juego y la inexistencia del recurso de apelación dentro del sistema CIADI, parecen agudizar aún más el problema. La solución no es fácilmente alcanzable: los Estados deben adoptar un papel más activo en la determinación del ámbito de aplicación de la CNMF, los árbitros deben interpretar el derecho y no crearlo, y el Centro debe tal vez estudiar la conveniencia de ciertas reformas.

\section{Referencias}

Ben Hamida, W. (2003). L'arbitrage EtatInvestisseur étranger. Réflexions sur une procédure réservée à l'initiative d'une personne privée contre une personne publique. Thèse pour le Doctorat en Droit de l'Université Panthéon-Assas (Paris II), 72-615.

Commission Du Droit International, CDI. (1974). Cinquième rapport sur la clause de la nation la plus favorisée - projet d'articles (articles 6 bis à 16). Document de travail, M. Endre Ustor, http://www.un.org/law/ french/ilc/index.htm

Commission Du Droit International, CDI. (1978). Projet d'articles sur les CNMF et commentaires. http://www.un.org/law/ french/ilc/index.htm

Convention de Vienne sur le Droit des Traités. (1969, 23 de mayo. Entrada en vigor 1980, 27 de enero). http://untreaty.un.org/ilc/texts/ instruments/francais/traites/ 1_1_1969_ francais.pdf. Viena.

Convention pour le Règlement des Différends Relatifs aux Investissements entre Etats et Ressortissants d'autres Etats. (Adoptée 1965, 18 mars. Et entrée en vigueur 1966, 14 octobre). http://icsid.worldbank.org. Washington.

Crepet Daigremont, C. (2006). Traitement national et traitement de la nation la plus favorisée dans la jurisprudence arbitrale récente relative à l'investissement international. En Anthemis (Ed.), Le contentieux arbitral transnational relatif aux investissements: nouveaux développements (pp. 107-162). Paris.

Crepet Daigremont, C. (2007). L'extension jurisprudentielle de la compétence des tribunaux du CIRDI. En Leiden (Ed), Les aspects nouveaux du droit des investissements internationaux, Recueil des cours de l'Académie de droit international de La Haye (vol. 328, pp. 453-516). Holanda: Martinus Nijhoff Publishers.

Dolzer, R., \& Myers T. (2004). After Tecmed: Most Favored Nation Clauses in Investment Protection Agreements. ICSID review: foering investment law journal, 19(1), 49-60.

Fietta, S. (2005). Most Favored Nation Treatment and Dispute Resolution under bilateral investment treaties: a turning point?. International Arbitration Law Review, 8, 131138.

FTAA, Acuerdo de Libre Comercio de las Américas - Borrador - (2003). Capítulo XVII. http:// www.ftaa-alca.org/FTAADraft03/TOC Word_s.asp 
Gaillard, E. (2005, 2 de junio). Establishing Jurisdiction through a Most-Favored-Nation Clause. NYLJ, 233. [www.nylj.com]

Gaillard, E. (2005). Note sous le Centre International de Règlement des Différends relatifs aux Investissements. Décision du 3 août 2004. Siemens AG c/ République d'Argentine. Journal de Droit International, 132, 142-163.

Gaillard, E. (2006). Note sous le Centre International de Règlement des Différends relatifs aux Investissements. Décision sur la compétence du 8 février 2005. Plama Consortium Limited c/ République de Bulgarie. Traité sur la Charte de l'Energie. Journal de Droit International, 133, 251-287.

Gaillard, E. (2007). Note sous le Centre International de Règlement des Différends relatifs aux Investissements. Sentence sur la compétence du 13 septembre 2006. Telenor Mobile Communications c/ République de Hongrie. Journal de Droit International, 134, 298-317.

Leben, C. (2006). L'évolution du droit international des investissements: un rapide survol. En Anthemis (Ed.), Le contentieux arbitral transnational relatif aux investissements : nouveaux développements (pp. 9-22). Paris. [Versión electrónica: http://www.dundee. ac.uk/cepmlp/journal/html/vol7/article712.html]

Nafta, North America Trade Agreement. (1994). Mexique, Etats Unis et Canada. www.nafta.org

Poulain, B. (2007). Clause de la Nation la Plus Favorisée et Clauses d'Arbitrage InvestisseurEtat : Est-ce la fin de la jurisprudence Maffezini. Bulletin ASA, 25, 279-301.

Sutton, S. (2005). Emilio Augustin Maffezini v. Kingdom of Spain and the ICSID SecretaryGeneral's Screening Power. Arbitration International, 21, 113-126.
Unctad. (2000). Admission et établissement des investissements, Collection consacrée aux problèmes relatifs aux accords internationaux d'investissements. New York-Genève.

Unctad. (2003). Dispute Settlement: Investor - State Series on issues in International Investment Agreements. http://www.unctad. org/en/docs/iteiit30_en.pdf

Vesel, S. (2001). Clearing a Path through a tangled jurisprudence: Most Favored Nation Clauses and Dispute Settlement Provisions in Bilateral Investment. YJIL, 32, 125-189.

DECISIONES DE LA CORTE INTERNACIONAL DE JUSTICIA. www.icj.cij.org

Caso Anglo-Iranian Oil Co. (R.-U. c. Iran), Sentencia del 22 de julio de 1952.

Caso Ambatielos (Grecia c. R.-U.), Sentencia del 1ero. de julio de 1952.

Caso relativo a los derechos de los nacionales de EE.UU. en Marruecos (EE.UU c. Marruecos), Sentencia del 27 de agosto de 1952.

DECISIONES CIADI www.worldbank.org/ $\mathrm{icsid} /$ cases

Emilio Agustin Maffezini c. Royaume d'Espagne. (ICSID Case No. ARB/97/7), Decisión sobre las excepciones a la jurisdicción del 25 de enero de 2000.

Técnicas Medioambientales TECMED S.A. c. Estados Unidos Méxicanos. (ICSID Case No. $\operatorname{ARB}(\mathrm{AF}) / 00 / 2)$, Laudo del 29 de mayo de 2003.

Siemens AG. c. República de Argentina (ICSID Case No.ARB/02/8), Laudo del 3 de agosto de 2004.

Salini Costruttori c. The Hashemite Kingdom of Jordan. (ICSID Case No. ARB/02/13), Laudo del 29 noviembre de 2004. 
Camuzzi International v. República de Argentina (ICSID Case No. ARB/03/7) Decisión sobre la jurisdicción del 10 de junio de 2005.

Gas Natural SDG, S.A. v. República de Argentina (ICSID Case No. ARB/03/10) Laudo del 17 de junio de 2005.

Plama Consortium Limited v. Republic of Bulgaria. (ICSID Case No. ARB/03/04). Laudo del 8 de febrero de 2005.

Telenor Mobile Communications AS v. République de Hongrie. (ICSID Case No. ARB/04/15), Laudo del 13 de septiembre de 2006.
Wintershall Aktiengesellschaft c. República de Argentina (ICSID Case No. ARB/04/14), Laudo del 8 de diciembre de 2008

TSA c. República de Argentica (ICSID Case No. ARB/05/5), Laudo del 19 de diciembre de 2008.

\section{Otras decisiones}

Cámara de Comercio de Estocolmo: RosInvestCo c/ UK Ltd. Fédération Russe, Institute d'Arbitrage de la Chambre de Commerce de Stockholm, Caso: SCC V079/ 2005, IIC315(2007), Laudo de octubre 2007, http:// ita.law.uvic.ca/documents/RosInvest jurisdiction_decision_200710001.pdf 